\title{
Respuesta fisiológica, cambios en la resistencia aeróbica y la composición en un grupo de adultos practicantes ocasionales de deporte
}

\author{
Physiological response, changes in the aerobic endurance and body composition in a group of adult people who practice \\ sports activities occasionally
}

\section{Resumen}

\author{
Hernando Mosso Sánchez
}

La práctica de actividades deportivas realizadas los fines de semana, en especial los días domingos y festivos, tienen una amplia acogida en nuestro medio. Este fenómeno se incrementó considerablemente con el surgimiento de las ciclovías, donde personas de todas las condiciones realizan recorridos a lo largo de diferentes vias en bicicleta o cubriendo los trayectos o caminando. En la actualidad se realizan sesiones de danza aeróbica en diferentes sitios de la ciudad, las cuales son orientadas por personas contratadas por el Instituto Distrital para la Recreación y el Deporte, IDRD.

Quienes realizan este tipo de actividades pueden clasificarse en dos grandes grupos: uno conformado por aquellos que tienen por rutina realizar una actividad fisica una vez a la semana, la cual es incorporada como parte de sus hábitos de vida. El otro lo constituyen quienes por periodos determinados participan en torneos deportivos, lo que hace que el tiempo de práctica se reduzca a la duración preestablecida del campeonato.

Se considera que el ejercicio fisico practicado en forma regular y orientado adecuadamente, mínimo tres veces por semana, tiene un efecto positivo sobre la salud: mejor funcionamiento cardiaco, reducción de lípidos en la sangre, mayor tolerancia a la glucosa. Por el contrario, las prácticas esporádicas, además de no incidir en las anteriores variables, parecen incrementar el riesgo de lesiones, que pueden ir desde leves molestias musculares hasta el desencadenamiento de fatales accidentes cardiacos.

Este estudio se propuso indagar, en una muestra definida de adultos practicantes ocasionales de deporte, los siguientes aspectos:

- Establecer si existen diferencias en la resistencia aeróbica entre los deportistas ocasionales y personas sedentarias de similares caracteristicas que los anteriores en cuanto a edad, actividad laboral, estado de salud y consumo de licor, tabaco u otras sustancias.

- Determinar los hábitos y costumbres de los participantes en cuanto a procesos de calentamiento, hidratación y desarrollo de su actividad.

- Indagar acerca de la morbi-mortalidad derivada de la realización de actividades fisicas cuando las mismas puedan ser atribuidas a falta de preparación o insuficiente adaptación al esfuerzo como puede ser el caso de los deportistas ocasionales.

- Evaluar posibles cambios en la composición corporal de quienes se vinculan a prácticas deportivas de manera ocasional.

Palabras clave: Deportista ocasional. Resistencia aeróbica. Lesión. Composición corporal.

\section{Abstract}

Practicing sports activities on weekends, specially on Sundays and holidays, has had a widely welcome in most people. These activities have increased considerably with the emergence of the ciclovias, where people of every kind use different ways for going biking, going running or walking. At present time, aerobic dance sessions are carrying out at different places in the city. These sessions are directed by trainers who are hired by the Instituto Distrital para la Recreación y el Deporte (IDRD).

People who do these activities are classified in two big groups. One group is formed by people who have a routine physical activity once a week, and this activity is incorporated into their life-style. The other group is formed by people who practice in sports tournament at specific period of time, and thus the training sessions are reduced to the pre-established duration of the championship. It is concluded that doing physical exercises regularly and oriented adequately at least three times a week has a very good effect on health: a better heart function, a lipid reduction in the blood, a great glucose tolerance. Otherwise, the sporadic training sessions seem to increase the damage risks, and these injuries may be so slight as muscular pains or so grievous as fatal heart disorders. This study was carried out on a specific sample of adults who practice sports activities occasionally, in order to investigate the following aspects:

- To establish if there are differences in aerobic endurance among occasional sportspeople and sedentary people in similar conditions as regards age, labor activity, health, and consumption of liquor, tobacco and other substances.

- To determine the participant's habits regarding warm-up and hydration processes, and activity development.

- To find out about the morbidity and mortality derived from carrying out physical activities when they may be attributed to lack of training or insufficient adaptation to the effort as it may be the case of occasional sportspeople.

- To evaluate possible changes in body composition of people who practice sports activities occasionally.

Key words: $\quad$ Body composition, aerobic endurace, physilogical response.

Fecha de recepción: 18 de marzo de 2005.

Fecha de aceptación: 12 de agosto de 2005

Médico, docente Universidad Pedagógica Nacional. 


\section{Antecedentes del problema}

Actualmente, en los países desarrollados menos del 1\% de la energía utilizada para la realización de tareas laborales proviene del hombre. En nuestro medio, si bien distamos mucho de la automatización generalizada de los procesos productivos, la tendencia generalizada de las diversas actividades nos conduce en esa dirección. En consecuencia, se ha incrementado un grupo de enfermedades conocidas como hipoquinéticas, cuya causa principal se atribuye al sedentarismo. Frente a esta situación se ha propugnado la realización de actividades físicas y deportivas de manera que las mismas compensen la necesidad de movimiento que tiene el hombre para mantener su organismo en óptimas condiciones. Esto, sin embargo, ha propiciado que muchas personas se lancen a realizar prácticas sin un conocimiento y una orientación adecuados en cuanto a la intensidad, volumen, precauciones y demás aspectos involucrados en el desarrollo de la actividad física. Claro que muy buena parte de quienes participan en las jornadas deportivas de manera ocasional no lo hacen pensando en la promoción de la salud; sus motivaciones tienen un carácter psicosocial, por cuanto a través de ellas moderan de alguna manera las tensiones generadas en su vida cotidiana, son una oportunidad para reunirse con sus amigos y, en general, constituyen un espacio de socialización y esparcimiento que no tiene más que ese propósito. Sin embargo, independientemente de la intención, es conveniente que se analicen los diversos elementos involucrados en el desarrollo de sus actividades a fin de detectar problemas y buscar la manera de hacerles llegar las recomendaciones que mejoran la calidad y el aprovechamiento de las mismas.

En 1996, Cafesalud planteó la necesidad de efectuar algunas valoraciones físicas entre los asistentes a las ciclovías, propósito que sería acompañado por el IDRD. Sin embargo, y por razones que se desconocen, el proyecto nunca se llevó a cabo.

Tomando en cuenta una amplia revisión bibliográfica y consultando en diferentes fuentes no se encontró ningún estudio que analizara el impacto que tiene la realización ocasional de deportes entre los adultos, por lo que éste constituye, hasta donde sabemos, la primera aproximación, a dicho fenómeno.

\section{Justificación}

Las enfermedades relacionadas con el sedentarismo han propiciado un creciente interés en la promoción de actividades físicas y deportivas. Sin embargo, algunas de es- tas prácticas carecen totalmente de orientación, control y seguimiento. Aunque en general se aconseja no practicar actividad física intensa sólo una vez por semana, no se conocen estudios que revelen la incidencia de ésta obre la morbi-mortalidad ni sobre el efecto fisiológico que las mismas pueden tener en quienes realizan éstas prácticas.

Con base en las consideraciones anteriores, creímos oportuno iniciar un estudio que establezca la realidad de estos aspectos para establecer las recomendaciones pertinentes e impulsar así a la Facultad de Educación Física de la Universidad Pedagógica Nacional(UPN) en el estudio de la población adulta practicante de deportes, y subsanar un descuido injustificado ante esta población.

\section{Objetivos}

a. Evaluar y contrastar la aptitud física de los deportistas ocasionales frente a un grupo con similares características de adultos sedentarios con el fin de comparar su evolución durante un periodo de ocho meses, para establecer las diferencias en el consumo máximo de oxígeno $\left(\mathrm{VO}_{2}\right)$ y la composición corporal entre los dos grupos.

b. Establecer la evolución de la aptitud física de los deportistas ocasionales mediante controles periódicos de la misma, y conocer sus principales hábitos en cuanto a calentamiento, hidratación y demás aspectos relevantes de su práctica.

c. Estudiar la morbi-mortalidad que pueda derivarse de la realización de actividades deportivas efectuadas en forma ocasional y sin la orientación adecuada, con el propósito de alentar la participación de la Facultad de Educación Física de la UPN ante las personas y entidades que promueven las prácticas físico-deportivas entre la población adulta a fin de que estas se realicen de manera más segura y saludable.

\section{Marco teórico}

Aptitud física. La definición de estos términos suscita sin duda controversia: ¿Aptitud para qué? ¿Cuál es el nivel adecuado de $\mathrm{VO}_{2}$, el grado de flexibilidad de los músculos isquiotibiales o la cantidad óptima de flexiones abdominales que una persona debe realizar? ¿Es importante que las personas incrementen sus parámetros de resistencia, fuerza y flexibilidad porque esto incidirá en su bienestar y su salud o porque los hará más eficientes para el aparato productivo? ¿O por qué de esta 
manera se acercarán más a los parámetros estéticos que la actual sociedad impone? Como el propósito de este trabajo no es profundizar sobre aspectos, recomendamos a Jean Jacques Barreau y J. J. Morne, quienes ofrecen una visión amplia y detallada sobre estos temas. En cualquier caso, es claro que el concepto de aptitud física es necesariamente relativo y obedece a las necesidades reales que en un momento dado un individuo en particular requiere para satisfacer las exigencias que su actividad laboral, recreativa o deportiva demanden. Sin embargo, y para los propósitos de este estudio, acogimos los criterios del Colegio Americano de Medicina Deportiva, que establecen valores mínimos de consumo de oxígeno $\left(\mathrm{VO}_{2}\right)$ para clasificar el nivel de aptitud de acuerdo con el sexo y la edad del evaluado. Ante la justificada crítica que puede hacerse frente a esta decisión, sólo podemos afirmar que en nuestro medio no hemos realizado estudios que establezcan dichos parámetros y justamente esta observación puede ser un intento por comenzar su realización.

La Federación Española de Medicina Deportiva recoge buena parte de los conceptos que actualmente predominan acerca de la actividad física y la salud:

\section{El ejercicio físico como un factor importante para la salud}

El cuerpo humano posee una gran capacidad de adaptación funcional y estructural al ejercicio físico enérgico. Los hombres fueron nómadas y cazadores durante miles de años de evolución. En los últimos tiempos se ha producido una reducción drástica de la cantidad de actividad física en la vida diaria, debido a los sistemas de ahorro de trabajo y al transporte motorizado.

Una consecuencia de esta disminución del ejercicio físico ha sido un descenso de la forma física en la población del mundo industrializado, con un aumento simultáneo del predominio de las enfermedades cardiovasculares como causa de muerte e incapacidad. (Segunda causa de mortalidad en $\mathrm{Co}-$ lombia después de la violencia)

Esto indica que el cambio a un estilo de vida sedentario puede ser perjudicial para el individuo y potencialmente costoso para la sociedad. Los estudios no han demostrado una relación directa entre la falta de ejercicio físico y la morbilidad y mortalidad cardiovasculares. Sin embargo, los datos epidemiológicos son claramente indicativos de los efectos beneficiosos del ejercicio físico en la prevención de las arteriopatías coronarias y en la disminución de la mortalidad por todas las causas cuando el ejercicio constituye una parte integrante de las activida- des laborales y recreativas. Además, al mejorar el perfil lipídico de la sangre, mantener la presión arterial dentro de límites seguros y controlar el peso corporal, el ejercicio físico puede modificar otros factores de riesgo. Por añadidura, el ejercicio puede contribuir al control de la diabetes méllitus y al mantenimiento de densidad ósea en el anciano.

Aunque la salud física, valorada por los índices de morbilidad y mortalidad, ha mejorado de modo constante en todo el mundo, los datos epidemiológicos y experimentales indican que es importante que la persona participe en un programa de ejercicio físico regular como parte de un estilo de vida sano. El seguimiento de un programa regular de ejercicio aerobio que movilice grandes grupos musculares puede lograr una potencialidad de los sistemas fisiológicos que mantienen esa actividad, y una mejoria simultánea de la capacidad para realizar ese ejercicio, y desembocar en el estado que recibe normalmente la denominación de forma fisica. Una persona en forma física tiene mayor capacidad para tolerar los desafíos físicos que plantea la vida diaria, mientras que la que no está en forma se verá obligada a interrumpir la actividad a causa de la fatiga.

La forma física y la buena salud no son sinónimas, pero si complementarias. Mientras que la buena salud significa simplemente ausencia de enfermedad, la forma física presupone energía suficiente para buscar las abundantes recompensas de la vida y no depender físicamente de otros. En la medicina deportiva se considera de importancia capital el problema de prevenir o remediar los efectos negativos de un estilo de vida sedentario y del envejecimiento. Por tanto, la actividad física adecuada constituye un valioso componente de los regímenes terapéuticos para el control y tratamiento de la cardiopatía coronaria, la hipertensión sistémica, la obesidad, los trastornos musculoesqueléticos, las enfermedades respiratorias y la depresión. La forma física puede aportar también una sensación de bienestar $\mathrm{y}$ autoestima.

\section{Metodología}

\section{Tipo de estudio}

Estudio de caso de tipo descriptivo, longitudinal, que permite establecer el nivel de resistencia aeróbica y los cambios en la composición corporal de individuos practicantes ocasionales de deportes, frente a otros predominantemente sedentarios. El estudio revelará, además, información sobre morbi-mortalidad registrada entre practicantes ocasionales de deporte. 


\section{Población de referencia}

La población de adultos entre los 35 y los 54 años de edad.

\section{Población de estudio}

Adultos practicantes ocasionales de deportes y/o sedentarios, comprendidos entre los 35 y los 54 años, que reunieran las siguientes características: no padecer ninguna enfermedad de tipo crónico, utilizar más del $75 \%$ de su tiempo laboral sin desplazarse, no consumir más de 10 cigarrillos diarios, no realizar actividades físicas o deportivas más de seis veces al mes, ingestión moderada de licor y no más de cuatro veces al mes, y finalmente disposición para participar en evaluaciones físicas bimensuales por un periodo de ocho meses. Se dispuso, además, la conformación de tres subgrupos, que cumplieran con los requisitos previamente mencionados, divididos de la siguiente manera:

Grupo A: practicantes regulares de actividad física los días domingos y festivos.

Grupo B: participantes en torneos deportivos para veteranos con actividad física sólo en el desarrollo de tales eventos.

Grupo C: grupo de sedentarios.

Se seleccionaron grupos específicos dadas las dificultades que hubiese implicado extraer una muestra representativa de un segmento tan grande de la población comprendida entre las edades escogidas. Para la conformación de los grupos se procedió a realizar entrevistas (Anexo 2) a adultos comprendidos entre las edades seleccionadas y que se encontraran practicando algún tipo de actividad física en el momento de ser abordados. Se realizaron en total 600 en los siguientes lugares: parque San Cristóbal, parque de Timiza, parque del Olaya, club Morato, de Telecom, parque Simón Bolívar, Cra. 50 con calle tercera. Una vez tabuladas las respuestas se escogieron sólo las personas que reunían los requisitos expuestos previamente, quedando el grupo reducido a 43 personas: 31 hombres y 12 mujeres. A todos se les citó para la explicación del proyecto; a esta instancia acudieron 28 personas: 19 hombres y 9 mujeres. Finalmente a la primera evaluación concurrieron 20 personas: 16 hombre ( $80 \%$ ) y 4 mujeres ( $20 \%)$, los cuales conformaron el grupo $\mathrm{A}$.

Para el grupo B se establecieron contactos con organizaciones de torneos de fútbol y microfútbol de categorías veteranos y mayores, especialmente intra e ínter empresas. Se distribuyeron 300 entrevistas entre quie- nes participaban de dichos eventos y se obtuvo un grupo inicial de 53 personas ( $100 \%$ hombres) contactados telefónicamente.

A la reunión explicativa acudieron 36 personas y a la valoración inicial 26.

El grupo C fue conformado por 18 personas (15 hombres y 3 mujeres), seleccionadas entre compañeros de trabajo de los grupos anteriores. La razón para incluirlos tomó en cuenta que: como cumplían funciones laborales similares, era de esperarse niveles de resistencia física que no se vieran especialmente afectados por el tipo de actividad que desempeñaban. Además, se estimulaba la asistencia a las evaluaciones al realizar las mismas entre grupos de compañeros.

\section{Duración: el estudio duró 12 meses:}

Marzo de 1999 a marzo de 2000.

\section{Evaluación de la aptitud física de los participantes}

A todos se les realizaron valoraciones antropométricas con el objeto de establecer índice de masa corporal y porcentaje de grasa.

La determinación de la resistencia aeróbica se estableció a través de la prueba de Rockport ${ }^{1}$; la cual consiste en caminar una milla $(1.609 \mathrm{~m})$ tan rápidamente como se pueda. Se toma en cuenta la frecuencia cardiaca al final y el tiempo que se ha tardado. Se ha desarrollado una ecuación de regresión que permite establecer el $\mathrm{VO}_{2}$ (Howley y Franks, 2001).

$\mathrm{VO}_{2} \max =132,6-\left(0,17^{\star} \mathrm{Pc}\right)-\left(0,39^{\star}\right.$ edad $)+6,31^{\star}$ sexo $)$ $-\left(3,27^{\star} \mathrm{T}\right)-\left(0,156^{\star} \mathrm{FC}\right)$

Donde: $\mathrm{VO}_{2} \max =\mathrm{Ml} \cdot \mathrm{Kg}^{-1} \cdot \mathrm{min}^{-1}$

$\mathrm{Pc}=$ peso corporal; sexo $=0$ mujeres, 1 hombre; edad $=$ edad en años; Tiempo = tiempo en minutos (00:00); FC $=$ frecuencia cardiaca.

Para la determinación del índice de masa corporal (IMC) se procedió a determinar estatura y peso. De donde $\mathrm{IMC}=$ Peso $/ \mathrm{H}^{2}$

Peso $=$ peso en kilogramos

$\mathrm{H}=$ estatura en metros

La determinación del porcentaje de grasa se realizó mediante la toma de tres pliegues cutáneos, los cuales permiten establecer la densidad corporal (Dc) (Pollock, Wilmore y Fox, 1990)

\section{Hombres}

$\operatorname{Dc}(3)=1.10938-(0.0008267 * 3)+\left(0.0000016^{*} 3\right)$

$-\left(0.00012828^{*}\right.$ edad $)$

$\mathrm{Dc}=$ Densidad corporal 
3 = suma de los pliegues cutáneos del pecho, abdomen y muslos $(\mathrm{mm})$ para los hombres y tríceps, suprailiaco y muslo para las mujeres. Para el porcentaje de grasa corporal se utiliza la ecuación de la densidad corporal, de Siri (Siri, 1961).

$\% \mathrm{Gc}=((4,91 / \mathrm{Dc})-4,50)^{\star} 100$

Las siguientes tablas establecen valores tanto para el $\mathrm{VO}_{2}$ (resistencia aeróbica) como para el IMC y porcentaje de grasa:

\begin{tabular}{|c|c|c|}
\hline $\begin{array}{c}\text { Clasificación } \\
\text { No obeso }\end{array}$ & Hombres & Mujeres \\
\hline $\begin{array}{c}\text { Moderadamente } \\
\text { obeso }\end{array}$ & $<25$ & $<27$ \\
\hline Obeso & $25-30$ & $27-30$ \\
\hline
\end{tabular}

Tabla 6.1. Normas de IMC (Di Girolamo, 1986).

\section{Porcentajes de grasa y su clasificación}

\section{Hombres}

\begin{tabular}{|c|c|c|c|c|c|}
\hline Edad & Ideal & Buena & Moderada & Sobrepeso & Obeso \\
\hline$<19$ & 12 & $12,5-17,0$ & $17,5-22,0$ & $22,5-27,0$ & $>27,5$ \\
\hline $20-29$ & 13 & $13,5-18,0$ & $18,5-23,0$ & $23,5-28,0$ & $>28,5$ \\
\hline $30-39$ & 14 & $14,5-19$ & $19,5-24,0$ & $24,5-29,0$ & $>29,5$ \\
\hline $40-49$ & 15 & $15,5-20,0$ & $20,5-25,0$ & $25,5-30,0$ & $>30,0$ \\
\hline $50>$ & 16 & $16,5-21,5$ & $22,0-26,0$ & $26,5-31,0$ & $>31,5$ \\
\hline
\end{tabular}

\section{Mujeres}

\begin{tabular}{|r|r|r|r|r|r|}
\hline$<19$ & 17 & $17,5-22,0$ & $22,5-27,0$ & $27,5-32,0$ & $>32,5$ \\
\hline $20-29$ & 18 & $18,5-23,0$ & $23,5-28$ & $28,5-33,0$ & $>33$ \\
\hline $30-39$ & 19 & $19,5-24,0$ & $24,5-29,0$ & $29,5-34,0$ & $>34,5$ \\
\hline $40-49$ & 20 & $20,5-25,0$ & $25,5-30,0$ & $30,5-35,0$ & $>35,5$ \\
\hline$>50$ & 21 & $21,5-26,5$ & $26,5-31,0$ & $31,5-36,0$ & $>36,5$ \\
\hline
\end{tabular}

Tabla 6.2. Porcentaje de grasa redondeado al $0.5 \%$ más próximo. Fuente. HOEGER, W. W. K. (1989).

\section{Diseño $\mathrm{y}$ fases}

Fase 1

En este periodo se obtuvo de manera directa la información de acuerdo con los siguientes procedimientos:

- Contactos con organizadores de torneos

- Realización de entrevistas

- Contactos telefónicos

- Definición de grupos de estudio
Fase 2

Una vez definidos los grupos se iniciaron las evaluaciones de los parámetros establecidos: medición indirecta de $\mathrm{VO}_{2}$, porcentaje de grasa e índice de masa corporal. Estas valoraciones se realizaron entre mayo de 1999 y febrero de 2000.

\section{Plan de recolección de la información}

Las evaluaciones las realizaron directamente y sobre terreno el investigador y los colaboradores. Se utilizaron los formularios ya indicados, y se crearon formatos de seguimiento (Anexo 3) para evaluar la evolución de los participantes.

La información obtenida se revisó para encontrar inconsistencias, errores y vacíos. Otra información recogida tuvo que ver con el surgimiento de patologías derivadas de las prácticas deportivas realizadas por los participantes en el estudio así como la averiguación acerca de la morbi-mortalidad encontrada sobre deportistas ocasionales entre las entidades responsables de la salud en Bogotá, al igual que entre un grupo de médicos generales.

Un tercer aspecto valorado lo constituyó la observación sobre hábitos de los participantes en cuanto a calentamiento y pautas de hidratación.

\section{Resultados y análisis}

Determinación de $\mathrm{Vo}_{2}$ max, composición y porcentaje de grasa de los participantes

Se analizaron un total de 64 personas, con edades comprendidas entre los 35 y los 54 años, con una media de 43,67 y una distribución de $90,62 \%$ de sexo masculino $(n=58)$ y de $9,3785 \%$ de sexo femenino $(n=6)$.

Las tablas $6.1,6.2$ y 6.3 indican los valores promedio de IMC, porcentaje de grasa y $\mathrm{VO}_{2}$ de los tres grupos analizados desde la primera hasta la última toma.

\begin{tabular}{|c|c|c|c|c|c|c|c|}
\hline Toma & $\begin{array}{c}\text { Promedio } \\
\text { de edad } \\
\text { (años) }\end{array}$ & $\begin{array}{c}\text { IMC } \\
\text { H }\end{array}$ & $\begin{array}{c}\text { IMC } \\
\text { M }\end{array}$ & $\begin{array}{c}\text { \%Grasa } \\
\text { corporal }\end{array}$ & \multicolumn{2}{|c|}{ VO $_{2}$ max } \\
\hline 1 & 45,3 & 23,2 & 24,2 & 18,25 & 23,6 & $\begin{array}{c}42,31 \\
(B)\end{array}$ & $\begin{array}{c}36,5 \\
(B)\end{array}$ \\
\hline 2 & 45,5 & 23,5 & 24,2 & 18,5 & 23,8 & $\begin{array}{c}42,33 \\
(B)\end{array}$ & $\begin{array}{c}37,1 \\
(B)\end{array}$ \\
\hline 3 & 45,7 & 23,4 & 24,3 & 18,4 & 23,1 & $\begin{array}{c}42,27 \\
(B)\end{array}$ & $\begin{array}{c}36,9 \\
(B)\end{array}$ \\
\hline 4 & 45,9 & 24,7 & 24,5 & 19,6 & 25,2 & $\begin{array}{c}41,21 \\
(B)\end{array}$ & $\begin{array}{c}35,1 \\
(B)\end{array}$ \\
\hline
\end{tabular}

Tabla 6.1. Valores del grupo A (practicantes regulares de actividad fisica domingos y festivos) valoración realizada en mayo, agosto y noviembre de 1999 y febrero de 2000. 


\begin{tabular}{|c|c|c|c|c|}
\hline Toma & $\begin{array}{c}\text { Promedio } \\
\text { de edad } \\
\text { (años) }\end{array}$ & IMC & $\begin{array}{c}\text { \% Grasa } \\
\text { corporal }\end{array}$ & VO $_{2}$ MAX \\
\hline 1 & 41,3 & 28,5 & 24,4 & $32,56(\mathbf{M})$ \\
\hline 2 & 41,5 & 28,9 & 25,1 & $32,77(\mathrm{M})$ \\
\hline 3 & 41,7 & 29,1 & 24,5 & $35,57(\mathbf{M})$ \\
\hline 4 & 41,9 & 30,2 & 25,7 & $33,8(\mathbf{M})$ \\
\hline
\end{tabular}

Tabla 6.2. Valores del grupo B (practicantes regularmente sedentarios que participan de manera ocasional en torneos deportivos).

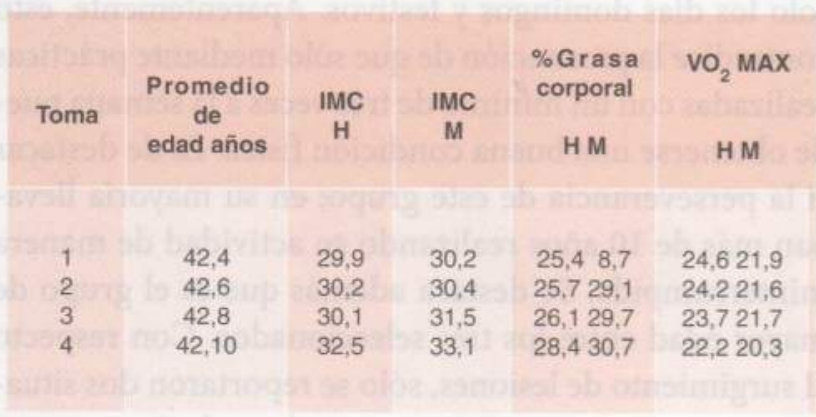

Tabla 6.3. Grupo $\mathrm{C}$ adultos sedentarios.

\begin{tabular}{|c|c|c|c|c|c|}
\hline \multicolumn{7}{|c|}{ Hombres } \\
\hline Edad & Baja & Regular & Media & Buena & Excelente \\
\hline$<29$ & $<24$ & $25-33$ & $34-42$ & $43-52$ & $>52$ \\
\hline $30-39$ & $<23$ & $25-30$ & $31-38$ & $39-48$ & $>48$ \\
\hline $40-49$ & $<20$ & $20-26$ & $27-35$ & $36-44$ & $>44$ \\
\hline $50-59$ & $<18$ & $18-24$ & $25-33$ & $34-42$ & $>42$ \\
\hline $60-69$ & $<16$ & $16-22$ & $23-30$ & $31-40$ & $>40$ \\
\hline & & & Mujeres & & \\
\hline$<29$ & $<24$ & $24-30$ & $31-37$ & $38-48$ & $>48$ \\
\hline $30-39$ & $<20$ & $20-27$ & $28-33$ & $34-44$ & $>44$ \\
\hline $40-49$ & $<17$ & $17-23$ & $24-30$ & $31-41$ & $>41$ \\
\hline $50-59$ & $<15$ & $15-20$ & $21-27$ & $28-37$ & $>37$ \\
\hline $60-69$ & $<13$ & $13-17$ & $18-23$ & $24-34$ & $>34$ \\
\hline
\end{tabular}

Tabla 6.4. Clasificación del $\mathrm{VO}_{2} \mathrm{MAX}$ de acuerdo con la edad y el sexo. Fuente: American Herat Association (1972).

De acuerdo con los resultados encontrados podemos destacar los siguientes hechos: el grupo A muestra valores de porcentaje de grasa y $\mathrm{VO}_{2}$ max considerados como buenos para la edad de los participantes. No se apreciaron cambios significativos en los elementos evaluados.

El grupo B mostró porcentajes de grasa ligeramente altos. En cuanto al $\mathrm{VO}_{2}$ se encontraron niveles medios de resistencia aeróbica, aunque vale la pena destacar una ligera evolución de la misma entre mayo y noviembre.

El grupo C reflejó porcentajes de grasa elevados y valores regulares de $\mathrm{VO}_{2}$; no se apreciaron cambios significativos en ninguno de los parámetros valorados.
Recolección de información sobre morbi-mortalidad y pautas de calentamiento e hidratación en deportistas ocasionales.

\section{Población estudiada}

En este punto se estuvo atento a recoger información sobre el surgimiento de lesiones que pudieran ser adjudicadas a la realización de las actividades deportivas, descartando aquellas derivadas de traumas directos provocados por terceros. Los participantes fueron instruidos para comunicar cualquier anomalía en sus condiciones de salud que en su opinión requiriera atención médica. De allí encontramos los siguientes resultados:

Grupo A. Durante los nueve meses de seguimiento se presentaron dos casos de esguince de tobillo entre quienes practicaban baloncesto. En ninguno de los dos casos las lesiones requirieron incapacidad o por lo menos así lo consideraron los médicos tratantes. No se reportó ningún otro caso entre este grupo.

Grupo B. En este grupo se presentó una pérdida temporal de conciencia, provocada por una baja de glicemia (nivel de glucosa en la sangre), según un seguimiento posterior. Además, dos casos de dolor retroesternal y disnea (síntomas de cardiopatía) surgidos en medio de los eventos deportivos (las cardiopatías fueron confirmadas posteriormente), doce caos de desgarros musculares y tres esguinces de cuello de pie.

Grupo C. No se registran casos, pues se trata de personas que no practican ninguna actividad deportiva.

Recolección sobre morbi-mortalidad en deportistasocasionales No fue posible obtener información epidemiológica a través de ninguna de las entidades públicas ni privadas consultadas. Se obtuvo sí información valiosa por parte del Instituto Nacional de Medicina Legal, del cual transcribimos:

Durante 1999, el Instituto de Medicina Legal y Ciencias Forenses de la regional Bogotá practicó necropsia médico legal a 599 personas que fallecieron de manera natural, de estas once (11) se encontraban practicando algún deporte en el momento de su muerte, según información aportada en el acta de inspección del cadáver.

Todos eran hombres, con edad promedio de 42,2 años (rango de 15 a 65 años) y la mayoría (4) de ellos estaban jugando fútbol, 3 practicando baloncesto, 2 montando bicicleta, 1 practicaba natación y otro estaba jugando tejo. El $100 \%$ de estas personas fallecieron por enfermedades cardiacas (arritmias cardiacas (3) infarto agudo del miocardio (4), otras cardiopatías (3) o ruptura de aneurisma de aorta (1), 
A nivel privado se creó un instrumento (núm. 4), el cual buscó establecer entre 100 médicos generales información sobre casos de muerte súbita, presencia de lesiones, factores predisponentes, lesiones más frecuentes y si recomendaban o no la práctica ocasional de deporte. Los resultados revelan lo siguiente:

a. El $60 \%$ conocía casos de muerte súbita ocurrida durante la práctica deportiva.

b. El $100 \%$ ha atendido casos de lesiones deportivas atribuibles a falta de preparación física.

c. En cuanto a los factores predisponentes las causas de las lesiones son atribuidas, en orden de importancia, a: falta de calentamiento, déficit de fortaleza muscular, incapacidad técnica.

d. Las lesiones más frecuentes son en su orden: desgarros musculares, luxaciones y esguinces, fracturas y alteraciones cardiovasculares.

\section{Información sobre hábitos de calentamiento e hidratación}

Para obtener esta información se acudió al diligenciamiento de un instrumento (anexo 1), el cual fue distribuido entre estudiantes de educación física. Se recogieron 120 formularios, que arrojaron la siguiente información:

La mayoría de quienes asisten a los diferentes parques realizan deportes de conjunto de manera informał (baloncesto y banquitas principalmente) sin realizar ningún tipo de calentamiento previo. Eventualmente ejecutan ciertos movimiento articulares o muy breves carreras en el puesto. Pero por lo general "calientan" a medida que desarrollan su juego. Tampoco se observó que se hidrataran previamente, lo que sí se hacían al final de la actividad, en especial con agua.

Entre quienes tienen la rutina de trotar alrededor de los parques, muy pocos realizaban estiramiento previo, pero calentaban durante el desarrollo propio de su actividad iniciando con ritmos ligeros, los cuales incrementaban de manera paulatina. Hidratación sólo al final.

Finalmente, los que participan en eventos deportivos organizados en las categorías de veteranos carecen de la cultura del calentamiento o lo hacen de manera muy superficial, tal como se describió con el primer grupo. Se apreció incluso que buena parte de los participantes se cambiaba mientras los árbitros hacían su ingreso al campo. La hidratación se hacía en el entretiempo con agua y en una alta proporción con cerveza al final de los encuentros.

\section{Análisis de resultados}

Composición corporal y $\mathrm{VO}_{2}$ max de los grupos estudiados e incidencia de lesiones.

El grupo A mostró porcentajes adecuados de grasa corporal, los cuales variaron de manera muy leve después del periodo navideño, (esto fue constante para los grupos). En cuanto a la resistencia aeróbica valorada a través del $\mathrm{Vo}_{2}$ apreciamos valores considerados como buenos de acuerdo con la Asociación Americana del Corazón, esto a pesar de que su actividad la realizan solo los días domingos y festivos. Aparentemente, esto contradice la presunción de que sólo mediante prácticas realizadas con un mínimo de tres veces a la semana puede obtenerse una buena condición física. Es de destacar sí la perseverancia de este grupo; en su mayoría llevaban más de 10 años realizando su actividad de manera ininterrumpida. Se destaca además que es el grupo de mayor edad entre los tres seleccionados. Con respecto al surgimiento de lesiones, sólo se reportaron dos situaciones que no necesariamente pueden relacionarse con preparación inadecuada, sino que son frecuentes en la práctica del baloncesto a cualquier nivel.

El grupo B mostró porcentajes ligeramente altos de grasa corporal y niveles medios de resistencia aeróbica. En este punto es preciso destacar que el deporte practicado (fútbol) por el grupo estudiado tiene un nivel de exigencia más o menos alto; que si bien no es constante, sí requiere un nivel adecuado de condición física, máxime si se toma en cuenta que ocasionalmente se precisan esfuerzos intensos, potencialmente peligrosos cuando no se está preparado para ellos. Con relación a las lesiones, llama la atención el elevado número de las mismas, pues de las 26 personas estudiadas, $18(69,2 \%)$ presentaron algún tipo de lesión que de alguna manera puede atribuirse a preparación física inadecuada. La pérdida de conciencia y la aparición de signos como dolor retroesternal y disnea son señales de graves trastornos de salud, los cuales si bien no pueden adjudicarse a la práctica deportiva, sí alertan sobre la necesidad de que quien participe en eventos de esta naturaleza acuda a una evaluación médica exhaustiva previa a su participación. Los desgarros musculares suelen presentarse cuando los músculos son sometidos a esfuerzos excesivos sin el adecuado descanso o cuando se realizan trabajos sin el calentamiento apropiado.

El grupo C mostró niveles moderadamente altos de porcentaje de grasa y bajos niveles de resistencia aeróbica, lo cual es compatible con su sedentarismo. 


\section{Conclusiones}

Como resultado del estudio podemos destacar varios aspectos:

a. El grupo A deja entrever que la práctica constante de actividad física, cuando se realiza durante periodos prolongados, tiene efectos positivos sobre la resistencia aeróbica, independientemente de la frecuencia semanal de la misma. Esto, por supuesto, no es absouto y puede obedecer a las condiciones particulares del grupo participante. Sin embargo, vale la pena subrayar que este grupo, que realiza ejercicio ocasional de manera regular, muestra buenos niveles de resistencia aeróbica, en contraste con los sedentarios.

b. Es importante destacar la estabilidad en la resistencia aeróbica, pues durante los meses del estudio no hubo variaciones significativas en el $\mathrm{VO}_{2}$ como suele suceder entre quienes inician un programa de acondicionamiento físico, en el que los cambios son significativos, en especial en de los primeros meses.

c. La participación en torneos deportivos de fin de semana no favorece la evolución física de quienes intervienen en ellos, aunque sí pueden incidir positivamente en aspectos psicosociales. Sin embargo, estos mismos efectos pueden mantenerse e incluso incrementarse si entre los organizadores se establecen controles previos, recomendaciones básicas y en general si se promueven campañas educativas que disminuyan la aparición de lesiones deportivas y prevengan situaciones, que como, vimos ponen en riesgo la propia vida de los participantes.

d. Merece atención especial el informe presentado por Medicina Legal, puesto que deja ver un porcentaje muy alto de caos de muerte súbita. Al observar el promedio de edad de los difuntos ( 42,2 años) se establece que eran personas en la plenitud de su vida productiva, que con certeza aún tendrían mucho que aportar a sus familias y a la sociedad. Es evidente, además, que desconocían la gravedad de sus problemas de salud. Esto nos lleva a insistir en la necesidad de realizar controles más rigurosos entre los organizadores de encuentros deportivos. $\mathrm{Al}$ respecto vemos con preocupación cómo, se promueve, incluso desde cajas de compensación, la vinculación a programas de acondicionamiento físico autodirigidos, bajo la modalidad de "con su firma se compromete a eximirnos de cualquier responsabilidad", como si sólo se tratara de un problema legal. Por ello, reiteramos e invitamos al Instituto Distrital de Recreación y Deporte, a las organizaciones promotoras del deporte empresarial, a las juntas de acción comunal y, por supuesto, a los médicos y educadores físicos a que de manera sostenida ofrezcan capacitación y generen conciencia entre los practicantes de deportes para que lo hagan de la manera más segura y saludable.

e. A pesar de no guardar una relación estrecha con el objeto de estudio de este trabajo, manifestamos nuestra inquietud respecto al precario sistema de recolección de datos sobre morbi-mortalidad, sin duda podemos afirmar que en aspectos de epidemiología tenemos serias deficiencias. Como resultado de ello no fue posible recoger información entre las entidades promotoras de salud. Sin embargo, la información recogida entre 100 médicos pone en evidencia que un importante porcentaje de ellos conocían casos de muerte súbita en escenarios deportivos, que las lesiones más frecuentes ocurrían básicamente por la ausencia de una cultura física suficientemente desarrollada, lo cual hace imperativo el fortalecimiento de la educación física como única vía para modificar de manera favorable esta situación. 


\section{Anexo 1. Ficha de observación}

Universidad Pedagógica Nacional Facultad de Educación Física. Ficha de observación del proyecto Respuesta fisiológica (cambios en el $\mathrm{VO}_{2} \max$ ) del adulto practicante ocasional de deporte

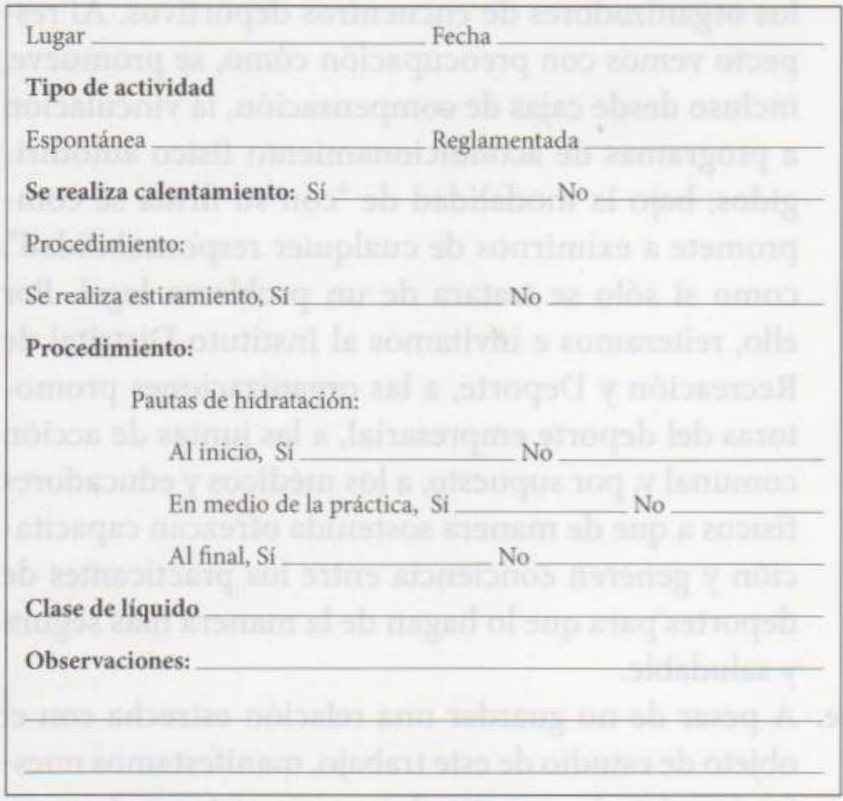

\section{Anexo 2. Ficha de información}

Universidad Pedagógica Nacional Facultad de Educación Física. Ficha de información sobre deportistas adultos (mayores 35 años) practicantes ocasionales de deportes.

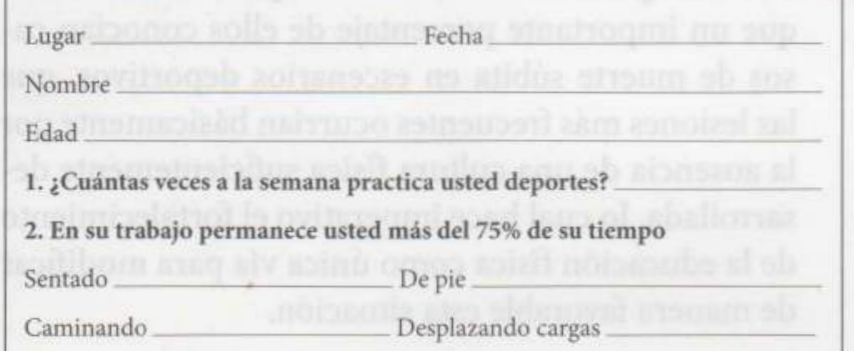

1. Fuma usted más de 10 cigarrillos diarios

Si No

2. Toma licor, $\mathrm{Si}$

Frecuencia mensual

3. Padece actualmente alguna enfermedad de tipo crónico

$\mathrm{Si}$ No

Toma algún medicamento

Si No

4. Estaría dispuesto a participar en un estudio, sobre condición física, en e que usted determinaria los dias y las horas de las evaluaciones No

5. En caso afirmativo anote su número telefónico
Anexo 3. Ficha de seguimiento

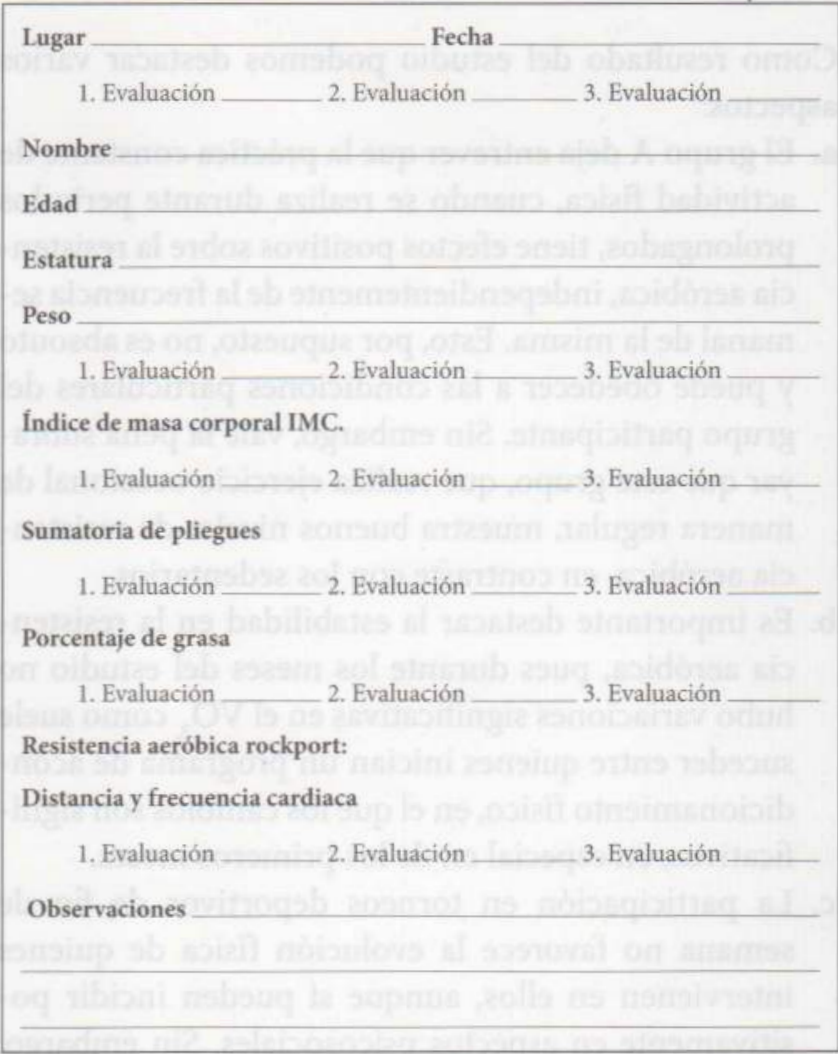

\section{Bibliografía}

BARREAU, Jean Jacques, MORNE, Jean J. (1991). Epistemologia y antropologia del deporte. Madrid. Alianza.

HOEGER, W. W. K. (1989). Lifetime physical fitness and wellness: a personalized program. Englenwood. Colorado. Morton Publishing Company, pp. 101-115.

HOWLEY, Edward y FRANKS, Don. (2001). Manual del técnico en salud y Fitness. Barcelona. Paidotribo.

MINISTERIO DE SALUD. Estudio sobre causas de morbi-mortalidad. En El Tiempo, febrero 23 de 2000. p. 2.

POLLOCK, K. W Wilmore, FOX and S. M. (1990). Exercise in Health an Disease. Evaluation and Prescription for Prevention and Rehabilitation. Philadelphia. Saunders College Publishing.

SERRA GRIMA, José Ricardo (1997). Prescripción del ejercicio para la salud. Madrid. Editorial Paidotribo.

SIRI, W. E. (1961). Body composition from fluid sauce and density: analysis of methots. In Brozek, and Henshel, A Eds. Techniques for Measurent Body Composition, pp 223-244. Washington, D.C., National academy of Sciences - National academy of Sciences-National Research Council.

\section{Notas}

1 Por imposibilidad logistica no se realizaron valoraciones directas del consumo de oxigeno, lo que hubiese sido deseable aunque no indispensable para los propósitos propuestos 